\title{
Focalidad Neurológica en Paciente Anticoagulado
}

\author{
Lorena López Martínez ${ }^{a}$, Francisco Hernández Fernández ${ }^{\text {, }}$ \\ Enrique Juliá Mollác, Ana Belén Perona Moratalla ${ }^{d}$
}

\begin{abstract}
a Médico Interno Residente del Servicio de Radiodiagnóstico del Complejo Hospitalario Universitario de Albacete.

${ }^{\mathrm{b}}$ Médico Interno Residente del Servicio de Neurología del Complejo Hospitalario Universitario de Albacete.
\end{abstract}

${ }^{\mathrm{c}}$ Médico Adjunto del Servicio de Radiodiagnóstico del Complejo Hospitalario Universitario de Albacete.

\section{${ }^{a}$ Médico Adjunto del Servicio de Neurología del Complejo Hospitalario Universitario de Albacete. \\ Correspondencia: \\ Lorena López Martínez, \\ Servicio de Radiodiagnóstico, \\ Complejo Hospitalario \\ Universitario de Albacete, \\ C/ Hermanos Falcó, s\n, 02006 \\ Albacete (España). \\ Telf.: 679846687, \\ e-mail: guati82@hotmail.com.}

Recibido el 16 de marzo de 2011.

Aceptado para su publicación el 25 de abril de 2011 .

\section{RESUMEN}

Presentamos el caso de una mujer de 63 años que acude a urgencias por dificultad en la emisión del lenguaje y debilidad en extremidades derechas de instauración súbita. Entre sus antecedentes personales encontramos una fibrilación auricular (FA) crónica insuficientemente anticoagulada. El primer diagnóstico a tener en cuenta es el de ictus isquémico agudo de origen cardioembólico. La enfermedad vascular cerebral es la segunda causa de mortalidad en el mundo. El tiempo entre el inicio del ictus y el tratamiento de revascularización, bien sea farmacológico (intravenoso o intraarterial) o mecánico nos marcará la evolución y pronóstico del paciente.

Palabras Clave. Accidente Cerebrovascular, Terapia Trombolítica, Procedimientos Endovasculares.

\section{ABSTRACT}

\section{Focal neurologic signs in an anticoagulated patient}

We report the case of a 63 year old woman who came to the emergency department due to difficulty in expressing herself linguistically and right limb weakness of sudden onset. She had a history of chronic atrial fibrillation (AF) and being insufficiently anticoagulated. The first diagnosis to consider is acute ischemic stroke of cardioembolic origin. Cerebrovascular disease is the second cause of death worldwide. The time between the onset of stroke and revascularization, either pharmacological (intravenous or intraarterial) or mechanical, will determine the patient's evolution and prognosis.

Key words. Stroke, Thrombolytic Therapy, Endovascular Procedures.

\section{INTRODUCCIÓN}

La enfermedad vascular cerebral es la segunda causa de mortalidad en el mundo, por detrás de la cardiopatía isquémica. Una TAC craneal urgente es una exploración indispensable en la evaluación inicial del paciente con ictus. El tiempo entre el inicio del ictus y el tratamiento de revascularización nos marcará la evolución y pronóstico del paciente. Por ello, se deberían realizar protocolos dentro de las estrategias del Código Ictus de los servicios de urgencias de nuestra región.

\section{OBSERVACIONES CLÍNICAS}

Mujer de 63 años sin alergias medicamentosas conocidas. Hipertensión arterial. Fibrilación auricular (FA) crónica anticoagulada. No antecedentes de epilepsia ni migrañas. No hábitos tóxicos. No intervenciones quirúrgicas previas. Tratamiento habitual con acenocumarol, bisoprolol, digoxina y torasemida.

Comienza a las 10:30 horas con dificultad para la emisión del lenguaje y debilidad en extremidades derechas de instauración abrupta. No fiebre, cefalea ni convulsiones. En los días previos no palpitaciones ni dolor torácico. Inicialmente es valorada en el centro de salud, que no activa Código Ictus por sospechar inicialmente una hemorragia cerebral. Tras 90 minutos de traslado es valorada en urgencias de su hospital de referencia. 
Exploración física: TA 152/83, FC 80 I.p.m, Tํㅡ 36,3ํㅡ, AC arrítmica, no se auscultan soplos ni extratonos. El resto de la exploración sistémica es normal. La exploración neurológica muestra una paciente en estado de alerta, con afasia global, desviación óculo-cefálica hacia la izquierda, reflejo de amenaza abolido en campo visual derecho, paresia facial supranuclear derecha, hemiparesia derecha (MSD 3/5, MID 0/5), impresiona de hipoestesia en extremidades derechas ante estímulos dolorosos y la marcha no es valorable. Presenta una puntuación de 24 en la National Institute of Health Stroke Scale (NIHSS).

En urgencias se realizó analítica con hemograma y bioquímica: sin alteraciones. En la coagulación: actividad de protrombina de $55 \%$ con INR de 1,4. ECG: FA a $60 \mathrm{lpm}$. Rx Tórax: sin hallazgos patológicos. Tomografía axial computarizada (TAC) cerebral urgente sin contraste: hiperdensidad de arteria cerebral media izquierda (ACMI) (figura 1,A), así como borramiento de la ínsula izquierda (figura 1,B). Doppler transcraneal (DTC) y cervical en fase aguda: ACMI con flujo muy amortiguado en M1. No se objetivan estenosis en ejes carotídeos.

Nos encontramos con una paciente de 63 años, con FA crónica insuficientemente anticoagulada y un déficit focal agudo de instauración súbita, lo cual, sumado a los hallazgos anteriormente nombrados, nos sugiere como primer diagnóstico a tener en cuenta el de ictus isquémico agudo de origen cardioembólico.

Aunque menos probable, se deben valorar otras entidades causantes de focalidad neurológica, como hematomas agudos, trombosis venosa, malformaciones arteriales, encefalitis infecciosas, tumores, vasoespasmo, vasculitis, crisis convulsivas o migrañosas. Para descartar algunas de ellas se debe solicitar un TAC de cráneo urgente.

Se activa Código Ictus y se traslada a nuestro centro. Es valorada por Neurología a las 4 horas y 15 minutos del inicio de los síntomas. Se decide tratamiento intraarterial ya que está fuera de protocolo para trombolisis intravenosa (más de 3 horas, INR 1.4). A las 5 horas del comienzo de los síntomas se realiza una arteriografía diagnóstica (figura 2) que muestra oclusión a nivel del tronco principal de la ACMI y de la trifurcación. Se realiza fibrinolisis intraarterial con r-TPA y posteriormente una perfusión continua de $9 \mathrm{ml}$ durante una hora en el trombo. El control final muestra recanalización completa de la ACMI.
La paciente ingresa en la Unidad de Ictus, iniciando tratamiento con perfusión IV de heparina sódica. A las 12 horas se realiza DTC donde se confirma la recanalización total de la arteria. La paciente muestra NIHSS 16 . Un nuevo TAC de control muestra hipodensidad parietal izquierda y borramiento de surcos cerebrales (figura $3, \mathrm{H}, \mathrm{I}$ ).

A las 24 horas comienza con heparina de bajo peso molecular y acenocumarol y se realiza un nuevo TAC que muestra una mejor definición del área infartada (figura $3, \mathrm{~J}$ ), con ganglios basales respetados.

La evolución durante el ingreso es satisfactoria. Al alta disfasia mixta de predominio motor, NIHSS 7. Se pauta anticoagulación oral con INR 2-3 de forma indefinida y seguimiento por parte de neurólogo y logopeda.

\section{COMENTARIOS}

La enfermedad vascular cerebral es la segunda causa de mortalidad en el mundo, situada sólo por detrás de la cardiopatía isquémica, y la primera causa de discapacidad. Además, su prevalencia está en aumento y produce elevadas tasas de recurrencia.

El ictus de origen cardioembólico es la segunda causa de ictus isquémico $(20-30 \%)$, siendo la segunda causa más frecuente, después del origen ateroesclerótico ${ }^{1}$. La FA es la causa más frecuente de ictus de causa embólica (50\%). Los infartos cardioembólicos suelen ser de tamaño medio o grande, y en general provocan mayor mortalidad y dependencia que los de otras etiologías ${ }^{1}$.

Los pacientes con embolias de origen cardíaco tienen una cierta ventaja frente a los de origen ateroesclerótico en el tratamiento con fibrinolíticos, ya que al ser de reciente formación y no estar firmemente adherido a la pared vascular, pueden fragmentarse con mayor facilidad.

EI TAC craneal es una exploración indispensable en la evaluación inicial del paciente con un ictus. Existen una serie de signos precoces que indican una lesión hiperaguda, como la hiperdensidad de la arteria y el borramiento de la ínsula o de los ganglios basales. Otros hallazgos como el borramiento de los surcos cerebrales y la pérdida de diferenciación sustancia gris, sustancia blanca indican una lesión aguda (hasta 12 horas de evolución) ${ }^{1}$. 
El pronóstico del paciente depende del tiempo entre el inicio del ictus y el tratamiento trombolítico con r-TPA, presentando una mejor evolución cuando este tiempo es menor ${ }^{2}$. El conocimiento de cómo el tiempo influye en el resultado clínico es crítico en la decisión del establecimiento de las terapias de revascularización. Sin embargo, la restauración del flujo sanguíneo cuando el daño es irreversible tiene mayor riesgo debido a las posibilidades de sangrado ${ }^{2}$.

Desde la introducción en la última década de las estrategias de Código Ictus y el tratamiento trombolítico dentro de una ventana terapéutica, el abordaje de la enfermedad cerebrovascular ha experimentado una revolución. El tratamiento con r-TPA en las 3 primeras horas ha demostrado ser un tratamiento efectivo, mejorando el pronóstico funcional a los 3 meses. A pesar del aumento de la tasa de hemorragias, el tratamiento ha demostrado su seguridad, ya que no hay un incremento significativo de la mortalidad ${ }^{3}$. Se han ido ampliando los esfuerzos para mejorar la ventana terapéutica y poder tratar pacientes más allá de las 3 horas. La publicación del ensayo ECAS-III, en $2008^{4}$, ha demostrado el beneficio clínico del tratamiento con r-TPA intravenoso entre las 3 y las 4,5 horas. $\mathrm{Se}$ registró un incremento significativo de las hemorragias post-tratamiento, sin empeorar la mortalidad. En ambos estudios se ha demostrado que el pronóstico empeora cuanto más se demore el inicio del tratamiento.

La recanalización depende del tamaño y localización del trombo. En nuestro caso, oclusión proximal de ACM, con trombolisis intravenosa se consigue aproximadamente un $30 \%$ de reperfusión ${ }^{5}$.

El abordaje intraarterial se emplea tradicionalmente cuando se rebasa la ventana terapéutica de la trombolisis intravenosa o cuando ésta ha sido inefectiva. Con elevadas concentraciones de trombolítico locales se amplía la ventana terapéutica a 6 horas y se alcanzan mejores datos de reperfusión precoz $(70 \%$ frente al $30 \%$ de la trombolisis intravenosa). La eficacia y seguridad de la trombolisis intraarterial dentro de las 6 primeras horas se ha demostrado en dos ensayos clínicos, PROACT I y $\|^{5}$.

El interés por ampliar la ventana terapéutica continúa incrementándose, pudiéndose emplear de forma efectiva dispositivos de extracción mecánica del coágulo hasta las 8 horas de clínica, como demostraron los ensayos $\mathrm{MERCl}$ y multi-
$\mathrm{MERCl}^{5}$. En el futuro dispondremos de mayor evidencia científica en este campo, con ensayos multicéntricos acerca de otros procedimientos (penumbra, stenting, angioplastia).

La clínica inicial de nuestra paciente es de un cuadro ictal. Para diferenciar un infarto hemorrágico de uno isquémico es imprescindible realizar unTAC cerebral. La presunción inicial de que se trataba de una hemorragia cerebral demoró de forma dramática el correcto manejo. Finalmente, la activación del Código Ictus y el acceso instantáneo a un tratamiento reperfusor intraarterial permitieron mejorar el pronóstico de la paciente.

En este caso existían varios factores que complicaban su abordaje. El tiempo de evolución límite (4 horas y 15 minutos) y la localización del coágulo (tronco de la ACMI) hacían que la trombolisis intravenosa presumiblemente tuviese una baja eficacia. Además, el elevado déficit neurológico y la toma previa de anticoagulantes orales incrementaban el riesgo de hemorragia.

Existe una sólida evidencia de la eficacia y seguridad del tratamiento reperfusor endovascular en el ictus agudo, por lo que creemos que debería incrementarse su disponibilidad y protocolizarse dentro de las estrategias de Código Ictus de los servicios de urgencias de nuestra región.

\section{BIBLIOGRAFÍA}

1. Bogousslavsky J, Cachin C, Regli F, Despland PA, Van Melle G, Kappenberger L. Cardiac sources of embolism and cerebral infarction. Clinical consequences and vascular concomitants: The Lausanne Stroke Registy. Neurology. $1991 ; 41: 855-959$.

2. Khatri P, Abruzzo T, Yeatts SD, Nichols C, Broderick JP, Tomsick TA. Good clinical outcome after ischemic stroke with successful revascularization is time-dependent. Neurology. 2009; 73:1066-72.

3. The National Institute of Neurological Disorders and Stroke rt-PA Stroke Study Group. Tissue plasminogen activator for acute ischemic stroke. N Engl J Med. 1995; 333(24):15817.

4. Hacke W, Kaste M, Bluhmki E, Brozman M, Dávalos A, Guidetti D, et al. Thrombolysis with alteplase 3 to 4.5 hours after acute ischemic stroke. N Engl J Med. 2008; 359(13):1317-29.

5. Meyers PM, Schumacher HC, Higashida RT, Barnwell SL, Creager MA, Gupta R, et al. Indications for the performance of intracranial endovascular neurointerventional procedures: a scientific statement from the American Heart Association Council on Cardiovascular Radiology and Intervention, Stroke Council, Council on Cardiovascular Surgery and Anesthesia, Interdisciplinary Council on Peripheral Vascular Disease, and Interdisciplinary Council on Quality of Care and Outcomes Research. Circulation. 2009; 119(16):223549. 


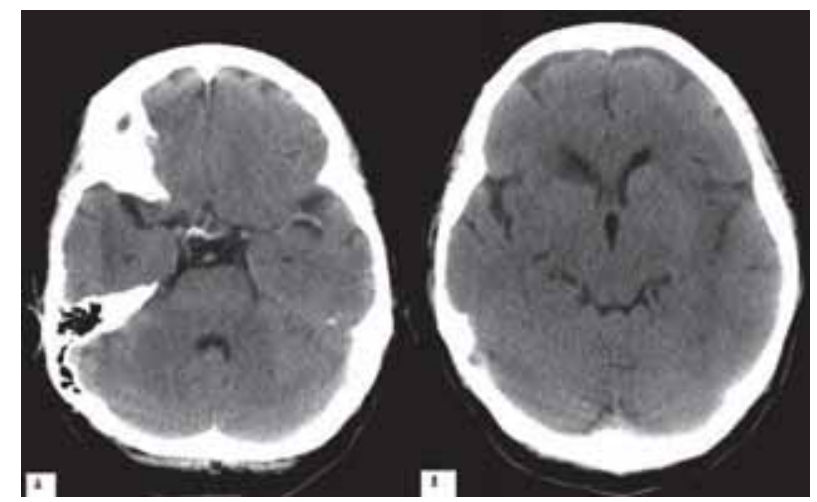

Figura 1. TAC cerebral sin contraste intravenoso. A. Se visualiza hiperdensidad de todo el trayecto de la ACM izquierda, lo cual indica un trombo de gran tamaño. B. Se objetiva un borramiento de la ínsula izquierda. Estos hallazgos son signos precoces de infarto a nivel de la ACM izquierda.

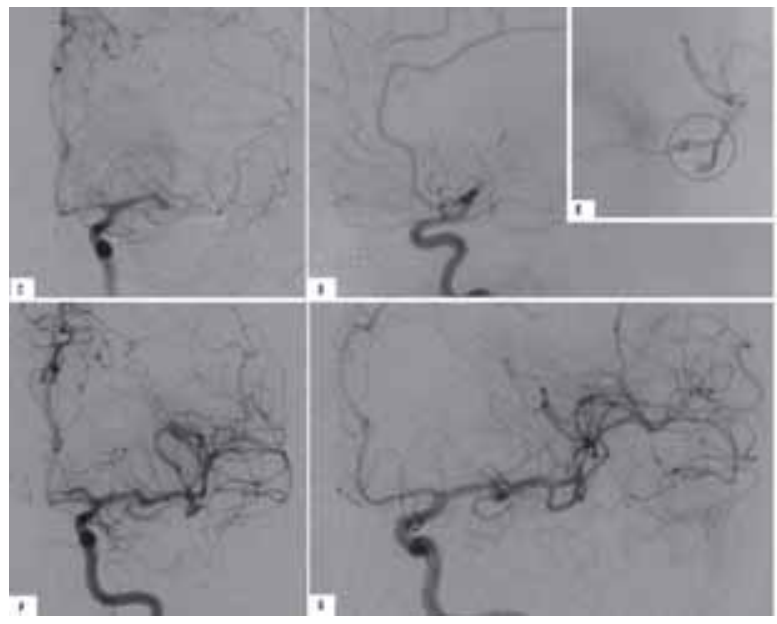

Figura 2. Arteriografía cerebral. TSA: Arteria carótida interna izquierda. C (proyección antero-posterior) y D (proyección lateral).

Oclusión de arteria cerebral media izquierda a nivel de porción M1. No hay paso de contraste distalmente. E. Defecto de repleción en relación con trombo en el interior de ACM izquierda. $\mathbf{F}$ (proyección antero-posterior) y $\mathbf{G}$ (proyección lateral). Recanalización completa de ACM izquierda tras el tratamiento intraarterial con el fibrinolítico.

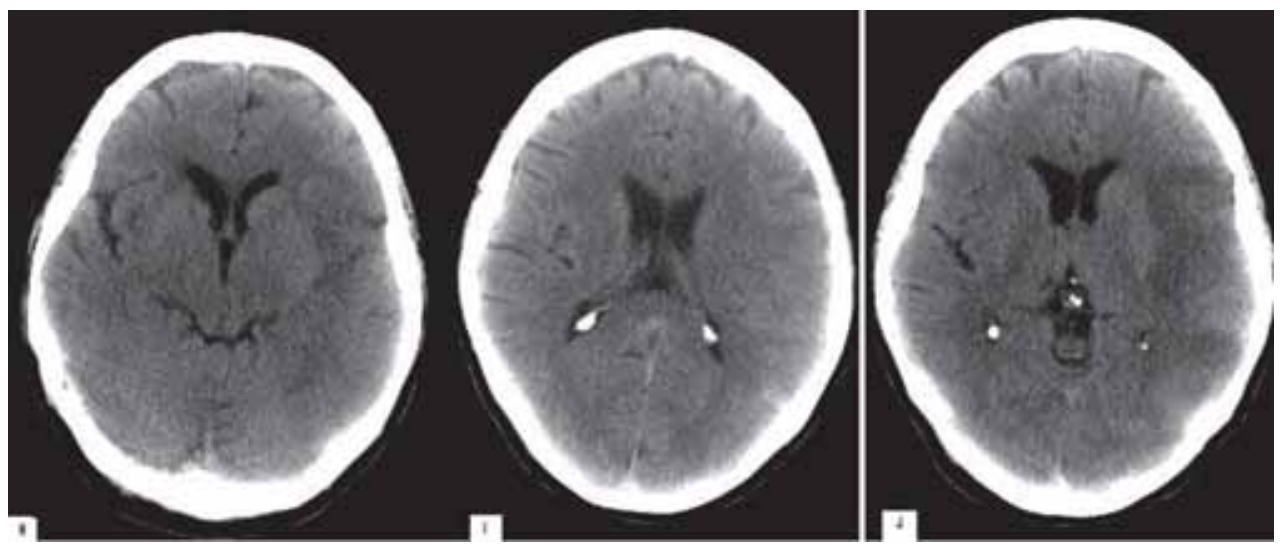

Figura 3. TAC cerebral de control 14 horas post-tratamiento. H. Área hipodensa parietal izquierda, subcortical, con afectación de ínsula ipsilateral y preservación de ganglios basales. I. Borramiento de surcos cerebrales parietales izquierdos. J. TAC cerebral sin contraste de control 24 horas post-tratamiento: área hipodensa parietal izquierda, cortico-subcortical, con borramiento de surcos cerebrales ipsilaterales. En ambos casos se muestra la evolución de la lesión isquémica de ACM izquierda, que respeta los ganglios basales ipsilaterales, probablemente debido a la eficacia del tratamiento. 